Article

\title{
The Impact of Smoking on the Association between Perfluoroalkyl Acids (PFAS) and Thyroid Hormones: A National Health and Nutrition Examination Survey Analysis
}

\author{
Maaike van Gerwen ${ }^{1,2, *}$, Naomi Alpert ${ }^{2,3}$, Mathilda Alsen ${ }^{1}$, Kimia Ziadkhanpour ${ }^{4}$, \\ Emanuela Taioli ${ }^{2,3,5}$ and Eric Genden ${ }^{1}$ \\ 1 Department of Otolaryngology-Head and Neck Surgery, Icahn School of Medicine at Mount Sinai, New York, \\ NY 10029, USA; mathilda.alsen@mountsinai.org (M.A.); Eric.Genden@mountsinai.org (E.G.) \\ 2 Institute for Translational Epidemiology, Icahn School of Medicine at Mount Sinai, New York, \\ NY 10029, USA; Naomi.alpert@mountsinai.org (N.A.); emanuela.taioli@mountsinai.org (E.T.) \\ 3 Department of Population Health Science and Policy, Icahn School of Medicine at Mount Sinai, New York, \\ NY 10029, USA \\ 4 Department of Medical Education, Icahn School of Medicine at Mount Sinai, New York, NY 10029, USA; \\ kimia.ziadkhanpour@icahn.mssm.edu \\ 5 Tisch Cancer Institute, Icahn School of Medicine at Mount Sinai, New York, NY 10029, USA \\ * Correspondence: maaike.vangerwen@mountsinai.org; Tel.: +1-212-659-9620; Fax: +1-212-423-2998
}

Received: 13 November 2020; Accepted: 7 December 2020; Published: 9 December 2020

\begin{abstract}
Perfluoroalkyl acids (PFAS) are known endocrine disrupting chemicals, potentially affecting thyroid function. Smoking has been associated with PFAS levels as well as with thyroid function. The impact of smoking on the association between PFAS and thyroid function remains to be elucidated, so the objective was to assess the effect of PFAS exposure on thyroid function in the general population, stratified by smoking status, using the National Health and Nutrition Examination Survey (NHANES). NHANES adult participants who were part of the 2011-2012 laboratory subsample and had PFAS and thyroid function measured were included $(n=1325)$. Adjusted linear regression models and stratified analyses were performed. There was a significant positive association between perfluorooctanesulfonic acid (PFOS) $(p=0.003)$, perfluorononanoic acid (PFNA) $(p=0.014)$, total PFAS $(p=0.004)$ concentrations and free T4 (FT4). No significant associations were found between perfluorooctanoic acid (PFOA), PFOS, perfluorohexane sulfonate (PFHxS), PFNA, total PFAS and total T4 (TT4) or thyroid stimulating hormone (TSH). In non-smokers, a significant positive association was found between PFOS $(p=0.003)$, PFHxS ( $p=0.034)$, PFNA $(p=0.012)$, total PFAS $(p=0.003)$ and FT4 while no significant associations were found in smokers. The present study showed that increased PFAS exposure was associated with increased FT4 in non-smokers, while no association was found in smokers. These results confirm that smoking modifies the association between PFAS exposure and thyroid function.
\end{abstract}

Keywords: thyroid; PFAS; effect modification; smoking

\section{Introduction}

Perfluoroalkyl acids (PFAS) are known endocrine disrupting chemicals with widespread persistence in the environment due to their stable chemical structure [1-3]. In addition to their presence in soil and water, PFAS have also been detected in the air and food, leading to an almost complete exposure of the general population (>95\%) [4]. In humans, the biological half-life for PFAS following absorption after inhalation and oral exposure is up to 8 years $[5,6]$. 
Due to their unique surfactant properties, PFAS were extensively used in surface coating and protectant formulations, including in paper and cardboard packaging products, carpets, leather products, firefighting foams, paints, and textiles in order to enhance water, grease, and soil repellency [7]. Since the early 2000s, when major American chemical companies voluntarily agreed to eliminate the use of perfluorooctanoic acid (PFOA) and PFOA-related chemicals, certain PFAS have been phased out and are no longer manufactured in the United States (US). Furthermore, the US Environmental Protection Agency (EPA) has established health advisory levels at 70 parts per trillion for PFOA and perfluorooctanesulfonic acid (PFOS) exposure from drinking water [8]. The European Union (EU) currently has restrictions on PFOS and PFOA under the EU Persistent Organic Pollutants (POPs) regulation (EU, 2019) and the Registration, Evaluation, Authorisation and Restriction of Chemicals (REACH) regulation (EU, 2006). The International Agency for Research on Cancer (IARC) listed PFOA as possibly carcinogenic (category 2B) to humans in 2017 [9]. Although restrictions are in place in the US and Europe, the use of PFOS has increased in China, which is problematic as PFAS are persistent in the environment and possibly transported over a long range [6].

PFAS have been identified as endocrine disrupting chemicals, potentially affecting the thyroid axis and thyroid function [10-16]. Higher concentrations of PFOA and PFOS were associated with thyroid disease in the general US population and in a large high exposure cohort $[17,18]$. Wen et al. found increased risk of subclinical hypothyroidism associated with PFAS exposure in men (PFOS) and women (PFOA, PFOS, perfluorohexane sulfonate (PFHxS)), but decreased risk of subclinical hypothyroidism after PFOA exposure in men [11]. Additionally, an association between PFOA exposure and hypothyroidism and a positive trend between estimated cumulative PFOA exposure and incident thyroid cancer was reported in a highly exposed community located near a chemical plant in the Mid-Ohio valley [18-20].

Prior studies have reported that there is an association between PFAS exposure and smoking status, with significantly higher levels of PFAS seen in smokers compared to non-smokers [21], as well as that smoking affects the thyroid gland. Active smoking was found to be associated with an inhibitory effect on the thyroid gland resulting in lower thyroid hormone levels in smokers compared to non-smokers [22]. Active smoking was also positively associated with mild thyroid stimulating hormone (TSH) decrease [23].

Although the association between PFAS exposure and thyroid function has been studied, the impact of smoking on this association remains an important topic to elucidate as no studies have been performed to date. The goal of the current study was therefore to assess the effect of PFAS exposure on thyroid function in the general population, stratified by smoking status, using the National Health and Nutrition Examination Survey (NHANES).

\section{Materials and Methods}

\subsection{Data Source and Study Population}

NHANES is a program designed to assess the health and nutritional status of the civilian, non-institutionalized population of the US and is administered by the National Center for Health Statistics. Since 1999, the program has been conducted continuously, with data released in a 2 year cycle. NHANES uses an ongoing, cross-sectional survey design, which combines questionnaires about demographics and health-related behaviors with physical examinations and laboratory tests. The NHANES program was approved by the National Center for Health Statistics Ethics Review Board (protocol \#2011-17, effective through 26 October 2017).

For this study, we queried NHANES participants, who were part of the 2011-2012 laboratory subsample. Only those with PFAS measurements and sample weights assigned $(n=2144)$ were included. We limited our study to participants 20 years and older, because information on the presence of thyroid disease and thyroid cancer is not available for younger participants $\left(n_{\text {exc }}=394\right)$. Participants were excluded if they were using thyroid medication $\left(n_{\text {exc }}=113\right)$, had thyroid disease 
or thyroid cancer $\left(n_{\text {exc }}=63\right)$, or were pregnant $\left(n_{\text {exc }}=28\right)$. The study population was further limited to participants who had thyroid function and PFAS measurements recorded $\left(n_{\text {exc }}=180\right)$. Lastly, we excluded participants with outlier thyroid function values of more than three standard deviations away from the mean $\left(n_{\text {exc }}=41\right)$, resulting in a final study population of 1325 participants. Covariates of interest included age, sex, race/ethnicity, body mass index (BMI), iodine status and self-reported smoking status.

\subsection{NHANES Laboratory Assessments}

Detection and quantification of serum PFAS concentrations was achieved using solid phase extraction coupled to high performance liquid chromatography/turbo ion spray ionization-tandem mass spectrometry (online SPE-HPLC-TIS-MS/MS), as described by Kuklenyik et al. [24]. A detailed description of the laboratory methods used can be found in the NHANES 2011-2012 Lab Methods for Polyfluoroalkyl Chemicals. Based on prior literature, we restricted our analysis to four PFAS commonly found in human sera, namely PFOA, PFOS, PFHxS, and perfluorononanoic acid (PFNA) [10,25]. The lower limits of detection (LLOD) for PFOA, PFOS, PFHxS and PFNA were $0.10,0.20,0.10$, and $0.08 \mathrm{ng} / \mathrm{mL}$, respectively. Values below that were imputed as LLOD/ $\sqrt{2}$, consistent with NHANES practice.

Although the NHANES thyroid profile consists of several thyroid function measurements, we restricted our analysis to total thyroxine (TT4), free thyroxine (FT4), total triiodothyronine (T3), free triiodothyronine (FT3) and thyroid stimulating hormone (TSH), as these measurements are referred to in guidelines to determine thyroid function [26]. Thyroid blood specimens were processed and stored at the Collaborative Laboratory Services, Ottumwa (Iowa); a detailed description of the laboratory methods used can be found in the NHANES 2011-2012 Laboratory Procedures Manual.

\subsection{Statistical Analysis}

In order to be representative of the population, NHANES uses a complex, multi-stage, probability sampling strategy. To account for this and produce nationally representative estimates, we used survey procedures and incorporated the survey design variables and weights. All analyses were performed using SAS software, version 9.4 (SAS Institute, Cary, NC, USA) and all statistics shown represent weighted values.

PFAS and thyroid function measurements were natural log (ln-) transformed. The association between PFAS levels and thyroid function measurements was examined using multivariable linear regression models with PFAS as a continuous predictor, adjusted for sex, race/ethnicity, age, BMI, iodine status and smoking status. Age was categorized into age groups (20-39 years, 40-59 years, and 60 years and older). BMI was categorized using the following cut-offs $<25 \mathrm{~kg} / \mathrm{m}^{2}, 25-30 \mathrm{~kg} / \mathrm{m}^{2}$ and $>30 \mathrm{~kg} / \mathrm{m}^{2}$, as proposed by the Center for Disease Control and Prevention (CDC). Following the World Health Organization's recommendations, iodine status was defined as normal ( $\geq 100 \mu \mathrm{g} / \mathrm{L}$ urine $)$ and low ( $<100 \mu \mathrm{g} / \mathrm{L}$ urine). Self-reported smoking status was categorized into smoker (current smoker) and non-smoker (never and former smoker). A sensitivity analysis was performed using serum cotinine levels, instead of self-reporting, to define smoking status. Cotinine, a metabolite of nicotine, is used as a biomarker to distinguish smokers from non-smokers in epidemiological studies [27]. Serum cotinine levels were used to classify smokers and non-smokers using cut off points based on race/ethnicity as described by Benowitz et al. [27]. We assessed the association between thyroid function measurements and the different PFAS (PFOA, PFOS, PFHxS, PFNA) as well as the total PFAS. The total PFAS level was calculated by summing the concentrations of the four separate compounds after converting to $\mu \mathrm{mol} / \mathrm{L}$ using molar weights, as described by Buttke et al. [28].

We then stratified the analysis by smoking status to analyze the effect of smoking on the association between PFAS and thyroid function measurements using self-reported smoking status. A sensitivity analysis was performed to test the robustness of the analysis by replacing the self-reported smoking status with serum cotinine levels. 


\section{Results}

Of the study population $(n=1325), 52.1 \%$ were male and $65.5 \%$ non-Hispanic white. Most participants were between 40 and 59 years old (40.1\%), followed by between 20 and 39 years old (38.2\%). Furthermore, $33.1 \%$ of the participants had a BMI $<25 \mathrm{~kg} / \mathrm{m}^{2}, 34.6 \%$ had a BMI of $25-30 \mathrm{~kg} / \mathrm{m}^{2}$ and $32.3 \%$ had a BMI of $>30 \mathrm{~kg} / \mathrm{m}^{2}$. Based on self-report, $20.6 \%$ of the sample were smokers, and $25.1 \%$ were smokers based on cotinine levels (Table 1 ).

Across the study population, geometric means (GMs; $\mu \mathrm{mol} / \mathrm{L}$ ) were $8.9,33.5,5.2$, and 4.3 for PFOA, PFOS, PFHxS, and PFNA, respectively. The GM for total PFAS for the study population was $55.2 \mu \mathrm{mol} / \mathrm{L}$, with a concentration of $67.5 \mu \mathrm{mol} / \mathrm{L}$ in males and $44.3 \mu \mathrm{mol} / \mathrm{L}$ in females. PFAS concentrations increased with age; 48.353 .5 , and $73.9 \mu \mathrm{mol} / \mathrm{L}$ for participants of 20-39 years, 40-59 years and 60 years and older, respectively. PFAS concentrations were $58.3 \mu \mathrm{mol} / \mathrm{L}$ and $44.6 \mu \mathrm{mol} / \mathrm{L}$ in non-smoker and smoker, respectively (Table 1). The mean TT4 and FT4 concentrations for the study population were $7.78 \mu \mathrm{g} / \mathrm{dL}$ and $0.82 \mathrm{ng} / \mathrm{dL}$, respectively. The mean TT3 and FT3 concentrations were $114.03 \mathrm{ng} / \mathrm{dL}$ and $3.17 \mathrm{ng} / \mathrm{mL}$, respectively; the GM for TSH was 1.50 milli-international units per liter (mIU/L). TSH levels increased with age from 1.42 and $1.47 \mathrm{mIU} / \mathrm{L}$ in 20-39 years and $40-59$ years old, to $1.72 \mathrm{mIU} / \mathrm{L}$ in 60 years and older (Table 1).

There was a significant positive association between PFOS $(p=0.003)$, PFNA $(p=0.014)$, total PFAS $(p=0.004)$ concentrations and FT4 and for PFNA $(p=0.024)$ concentration and FT3. No significant associations were found between PFOA, PFOS, PFHxS, PFNA, total PFAS and TT4 or TSH (Table 2). No interaction was found between PFAS and smoking $(p=0.211)$. Sensitivity analysis adjusting for smoking using serum cotinine showed the same results with a significant positive association for PFOS $(p=0.003)$, PFNA $(p=0.010)$, total PFAS $(p=0.004)$ concentrations and FT4 and for PFNA $(p=0.027)$ concentration and FT3.

A significant positive association was found between PFOS $(p=0.003), \operatorname{PFHxS}(p=0.034)$, PFNA $(p=0.012)$, total PFAS $(p=0.003)$ and FT4 in non-smokers while no significant associations were found in smokers (Table 3). A significant positive association was found between PFOA $(p=0.021)$ and PFNA $(p=0.015)$ and TT3 in smokers while no significant association was found in non-smokers (Table 3). Sensitivity analysis using serum cotinine levels showed similar results with a significant positive association between PFOS ( $p=0.002)$, PFHxS $(p=0.040)$, PFNA $(p=0.026)$, total PFAS $(p=0.003)$ and FT4 in non-smokers while no significant associations were found in smokers; however, no significant association was found between PFOA $(p=0.083)$, PFNA $(0.172)$ and TT3 in smokers. 
Table 1. Serum perfluoroalkyl acids (PFAS) and thyroid hormone concentrations by participant characteristics in study population $(n=1325)$.

\begin{tabular}{|c|c|c|c|c|c|c|c|c|c|c|c|}
\hline Variables & Total Population & PFOA $(\mu \mathrm{mol} / \mathrm{L})$ & PFOS $(\mu \mathrm{mol} / \mathrm{L})$ & PFHxS $(\mu \mathrm{mol} / \mathrm{L})$ & PFNA $(\mu \mathrm{mol} / \mathrm{L})$ & Total PFAS $(\mu \mathrm{mol} / \mathrm{L})$ & Free $\mathrm{T} 4(\mathrm{ng} / \mathrm{dL})$ & Total T4 $(\mu \mathrm{g} / \mathrm{dL})$ & Free T3 $(\mathrm{pg} / \mathrm{mL})$ & Total T3 (ng/dL) & $\mathrm{TSH}(\mathrm{mIU} / \mathrm{L})$ \\
\hline & $\mathrm{n}(\%)$ & GM (SE) & GM (SE) & GM (SE) & GM (SE) & GM (SE) & Mean (SE) & Mean (SE) & Mean (SE) & Mean (SE) & GM (SE) \\
\hline All & $1325(100)$ & $8.89(0.31)$ & 33.52 (1.13) & $5.20(0.26)$ & $4.26(0.19)$ & $55.18(1.65)$ & $0.82(0.01)$ & $7.78(0.08)$ & $3.17(0.03)$ & $114.03(1.26)$ & $1.50(0.03)$ \\
\hline \multicolumn{12}{|l|}{ Sex } \\
\hline Male & $717(52.1)$ & $10.13(0.37)$ & 42.22 (1.91) & $6.91(0.39)$ & $4.54(0.27)$ & $67.52(2.77)$ & $0.82(0.01)$ & $7.60(0.09)$ & $3.26(0.03)$ & $115.14(1.82)$ & $1.53(0.04)$ \\
\hline Female & 608 (47.9) & $7.71(0.38)$ & $26.07(1.01)$ & $3.81(0.23)$ & $3.99(0.14)$ & 44.29 (1.45) & $0.82(0.01)$ & $7.97(0.10)$ & $3.08(0.02)$ & $112.82(1.11)$ & $1.47(0.04)$ \\
\hline \multicolumn{12}{|l|}{ Age (years) } \\
\hline $20-39$ & $510(38.2)$ & $8.18(0.38)$ & $28.70(1.55)$ & $4.84(0.30)$ & $3.95(0.20)$ & $48.27(2.43)$ & $0.83(0.01)$ & $7.78(0.10)$ & $3.31(0.03)$ & $120.26(1.30)$ & $1.42(0.04)$ \\
\hline $40-59$ & $442(40.1)$ & $8.76(0.47)$ & 32.37 (1.12) & $4.80(0.29)$ & $4.19(0.27)$ & $53.50(1.81)$ & $0.80(0.01)$ & $7.68(0.10)$ & $3.16(0.03)$ & $112.19(1.57)$ & $1.47(0.08)$ \\
\hline$\geq 60$ & $373(21.7)$ & $10.58(0.61)$ & $46.97(2.46)$ & $6.81(0.52)$ & $5.04(0.30)$ & 73.89 (3.57) & $0.84(0.01)$ & $7.95(0.13)$ & $2.96(0.02)$ & $106.48(1.57)$ & $1.72(0.09)$ \\
\hline \multicolumn{12}{|l|}{ Race } \\
\hline NHW & 469 (65.5) & $9.68(0.46)$ & $35.66(1.32)$ & $5.97(0.36)$ & $4.20(0.25)$ & $58.80(2.11)$ & $0.81(0.01)$ & $7.67(0.10)$ & $3.16(0.03)$ & $112.82(1.69)$ & $1.56(0.05)$ \\
\hline NHB & 344 (11.5) & $7.66(0.28)$ & $34.17(3.07)$ & $4.49(0.20)$ & $4.55(0.26)$ & $54.26(3.76)$ & $0.82(0.01)$ & $8.01(0.19)$ & $3.16(0.02)$ & $115.49(2.24)$ & $1.25(0.04)$ \\
\hline Hispanic/other & $512(23)$ & $7.52(0.45)$ & $27.85(2.21)$ & $3.78(0.30)$ & $4.31(0.32)$ & $46.44(3.27)$ & $0.84(0.01)$ & $7.96(0.08)$ & $3.22(0.02)$ & $116.76(1.33)$ & $1.47(0.04)$ \\
\hline \multicolumn{12}{|l|}{ BMI $\left(\mathrm{kg} / \mathrm{m}^{2}\right)$} \\
\hline$<25$ & $446(33.1)$ & $8.85(0.42)$ & 32.75 (1.46) & $5.19(0.39)$ & $4.16(0.20)$ & $54.32(2.28)$ & $0.83(0.01)$ & $7.56(0.11)$ & $3.17(0.04)$ & $110.89(1.62)$ & $1.44(0.04)$ \\
\hline $25-30$ & 428 (34.6) & $9.88(0.60)$ & $36.36(2.02)$ & $5.86(0.39)$ & $4.80(0.26)$ & $60.64(2.82)$ & $0.81(0.01)$ & $7.79(0.10)$ & $3.15(0.03)$ & $114.87(1.52)$ & $1.54(0.05)$ \\
\hline$\geq 30$ & $451(32.3)$ & $7.98(0.35)$ & $31.46(2.10)$ & $4.58(0.34)$ & $3.84(0.23)$ & $50.67(3.03)$ & $0.81(0.01)$ & $7.98(0.11)$ & $3.20(0.03)$ & $116.36(1.84)$ & $1.51(0.05)$ \\
\hline \multicolumn{12}{|l|}{ Smoking* } \\
\hline Non-smoker & 1058 (79.4) & $9.30(0.33)$ & $35.61(1.27)$ & $5.38(0.28)$ & $4.47(0.20)$ & $58.33(1.86)$ & $0.82(0.01)$ & $7.79(0.09)$ & $3.16(0.02)$ & $113.11(1.30)$ & $1.52(0.04)$ \\
\hline Smoker & $266(20.6)$ & $7.50(0.51)$ & 26.56 (1.74) & $4.58(0.35)$ & $3.57(0.25)$ & $44.63(2.74)$ & $0.82(0.01)$ & $7.73(0.15)$ & $3.24(0.05)$ & 117.57 (1.92) & $1.41(0.07)$ \\
\hline \multicolumn{12}{|c|}{ Serum cotinine $(\mathrm{ng} / \mathrm{mL})$} \\
\hline Non-smoker & 993 (74.9) & $9.28(0.33)$ & $35.36(1.10)$ & $5.35(0.29)$ & $4.47(0.04)$ & $58.04(1.64)$ & $0.82(0.01)$ & $7.78(0.09)$ & $3.15(0.02)$ & $112.91(1.39)$ & $1.54(0.03)$ \\
\hline Smoker & $332(25.1)$ & $7.82(0.43)$ & $28.57(1.90)$ & $4.78(0.29)$ & $3.70(0.24)$ & $47.43(2.71)$ & $0.82(0.01)$ & $7.78(0.14)$ & $3.24(0.05)$ & $117.37(1.76)$ & $1.38(0.07)$ \\
\hline
\end{tabular}

* Questionnaire data (one missing); BMI: body mass index; GM: geometric mean; NHW: non-Hispanic white; NHB: non-Hispanic black; SE: standard error. 
Table 2. Weighted adjusted * analysis model estimates for the association between PFAS and thyroid hormones $(n=1325)$.

\begin{tabular}{|c|c|c|c|c|c|c|c|c|c|c|}
\hline & Ln TSH (mIU/L) & & Ln Total T4 $(\mu \mathrm{g} / \mathrm{dL})$ & & Ln Free T4 (ng/dL) & & Ln Total T3 (ng/dL) & & Ln Free T3 $(\mathrm{pg} / \mathrm{mL})$ & \\
\hline PFAS & $B_{a d j} *(95 \% C I)$ & $p$-Value & $B_{a d j} *(95 \% C I)$ & $p$-Value & $B_{a d j} *(95 \% C I)$ & $p$-Value & $B_{a d j} *(95 \% C I)$ & $p$-Value & $B_{a d j} *(95 \% C I)$ & $p$-Value \\
\hline Ln PFOA $(\mu \mathrm{mol} / \mathrm{L})$ & $0.006(-0.041 ; 0.053)$ & 0.787 & $-0.008(-0.027 ; 0.012)$ & 0.411 & $0.007(-0.007 ; 0.021)$ & 0.311 & $0.011(-0.009 ; 0.031)$ & 0.252 & $0.003(-0.011 ; 0.018)$ & 0.612 \\
\hline Ln PFOS $(\mu \mathrm{mol} / \mathrm{L})$ & $-0.002(-0.055 ; 0.051)$ & 0.943 & $-0.010(-0.024 ; 0.004)$ & 0.138 & $0.021(0.008 ; 0.035)$ & 0.003 & $0.001(-0.015 ; 0.017)$ & 0.873 & $0.002(-0.006 ; 0.010)$ & 0.565 \\
\hline Ln PFHxS $(\mu \mathrm{mol} / \mathrm{L})$ & $0.002(-0.036 ; 0.040)$ & 0.899 & $-0.005(-0.023 ; 0.013)$ & 0.558 & $0.011(-0.003 ; 0.025)$ & 0.127 & $0.010(-0.006 ; 0.026)$ & 0.198 & $-0.001(-0.015 ; 0.012)$ & 0.834 \\
\hline Ln PFNA $(\mu \mathrm{mol} / \mathrm{L})$ & $-0.005(-0.050 ; 0.041)$ & 0.837 & $0.000(-0.019 ; 0.020)$ & 0.968 & $0.021(0.005 ; 0.038)$ & 0.014 & $0.013(-0.006 ; 0.032)$ & 0.174 & $0.012(0.002 ; 0.023)$ & 0.024 \\
\hline Ln total PFAS $(\mu \mathrm{mol} / \mathrm{L})$ & $-0.004(-0.056 ; 0.049)$ & 0.883 & $-0.010(-0.027 ; 0.007)$ & 0.216 & $0.023(0.009 ; 0.038)$ & 0.004 & $0.003(-0.014 ; 0.020)$ & 0.716 & $0.003(-0.008 ; 0.014)$ & 0.613 \\
\hline
\end{tabular}

${ }^{*}$ Adjusted for sex, race, age group, BMI group, iodine status and smoking status (questionnaire); CI: confidence interval, Ln: Natural log transformed; Bold means significant.

Table 3. Stratified weighted adjusted * analysis model estimates for the association between PFAS and thyroid hormones in smokers and non-smokers $(n=1325)$.

\begin{tabular}{|c|c|c|c|c|c|c|c|c|c|c|}
\hline Smoking Status & Ln TSH (mIU/L) & & Ln Total T4 $(\mu \mathrm{g} / \mathrm{dL})$ & & Ln Free T4 (ng/dL) & & Ln Total T3 (ng/dL) & & Ln Free T3 (pg/mL) & \\
\hline Non-Smoker & $\mathrm{B}_{\mathrm{adj}} *(95 \% \mathrm{CI})$ & $p$-Value & $\mathrm{B}_{\mathrm{adj}} *(95 \% \mathrm{CI})$ & $p$-Value & $\mathrm{B}_{\mathrm{adj}} *(95 \% \mathrm{CI})$ & $p$-Value & $B_{a d j} *(95 \% C I)$ & $p$-Value & $B_{a d j} *(95 \% C I)$ & $p$-Value \\
\hline Ln PFOA $(\mu \mathrm{mol} / \mathrm{L})$ & $-0.001(-0.050 ; 0.049)$ & 0.975 & $-0.007(-0.025 ; 0.011)$ & 0.435 & $0.010(-0.005 ; 0.025)$ & 0.179 & $0.007(-0.015 ; 0.029)$ & 0.494 & $0.003(-0.013 ; 0.020)$ & 0.658 \\
\hline & $0.023(-0.067 ; 0.021)$ & & $0.010(-0.024 ; 0.005)$ & & $0.024(0.009 ; 0.039)$ & 0.003 & $-0.006(-0.021 ; 0.00)$ & 0.447 & $0.009 ; 0.008)$ & 0.840 \\
\hline & & & & & & & & & & 0.994 \\
\hline Ln PFNA $(\mu \mathrm{mol} / \mathrm{L})$ & $0.005(-0.047 ; 0.056)$ & 0.855 & $0.002(-0.02$ & 0.866 & $0.026(C$ & 0.0 & 0.007 & 0.50 & $0.013(-0.00$ & 0.094 \\
\hline Ln PFAS $(\mu \mathrm{mol} / \mathrm{L})$ & $-0.021(-0.066 ; 0.025)$ & 0.351 & $-0.010(-0.025 ; 0.006)$ & 0.205 & $0.026(0.010 ; 0.043)$ & 0.003 & $-0.004(-0.021 ; 0.013)$ & 0.645 & $0.000(-0.011 ; 0.012)$ & 0.938 \\
\hline Smoker & $\mathrm{B}_{\mathrm{adj}} *(95 \% \mathrm{CI})$ & $p$-Value & $\mathrm{B}_{\mathrm{adj}} *(95 \% \mathrm{CI})$ & $p$-Value & $\mathrm{B}_{\mathrm{adj}} *(95 \% \mathrm{CI})$ & $p$-Value & & & & \\
\hline Ln PFOA $(\mu \mathrm{m}$ & $.051(-0.073 ; 0.175)$ & 396 & $-0.016(-0.058 ; 0.025)$ & 0.424 & $-0.013(-0.042 ; 0.017)$ & 0.385 & & 0.021 & & 0.904 \\
\hline Ln PFOS $(\mu \mathrm{mol} / \mathrm{L})$ & $0.087(-0.041 ; 0.214)$ & & $-0.016(-0.057 ; 0.025)$ & 0.413 & $0.004(-0.021 ; 0.028)$ & 0.746 & $0.032(-0.002 ; 0.065)$ & 0.064 & $0.013(-0.007 ; 0.034)$ & 0.180 \\
\hline Ln PFHxS $(\mu \mathrm{mol} / \mathrm{L})$ & $0.074(-0.033 ; 0.180)$ & & $-0.019(-0.062 ; 0.024)$ & 0.365 & $-0.005(-0.035 ; 0.025)$ & 0.707 & $0.015(-0.023 ; 0.052)$ & 0.413 & $-0.003(-0.029 ; 0.022)$ & 0.786 \\
\hline Ln PFNA $(\mu \mathrm{mol} / \mathrm{L})$ & $-0.023(-0.129 ; 0.082)$ & & $-0.008(-0.048 ; 0.031)$ & 0.661 & & 0.726 & & 0.015 & $0.009(-0.007 ; 0.026)$ & 0.251 \\
\hline Ln PFAS $(\mu \mathrm{mol} / \mathrm{L})$ & $0.074(-0.055 ; 0.203)$ & 0.244 & $-0.020(-0.066 ; 0.027)$ & 0.389 & $0.003(-0.023 ; 0.029)$ & 0.808 & $0.033(-0.000 ; 0.067)$ & 0.054 & $0.011(-0.012 ; 0.034)$ & 0.333 \\
\hline
\end{tabular}

* Adjusted for sex, race, age group, BMI group, and iodine status; CI: confidence interval, Ln: Natural log transformed; Bold means significant. 


\section{Discussion}

The present study is the first study to investigate the effect of smoking on the association between PFAS exposure and thyroid function showing that increased PFAS exposure was associated with increased FT4 in non-smokers, while no association was found in smokers. These results confirm that smoking modifies the association between PFAS exposure and thyroid function, underlining that this is an important issue to be addressed in future studies.

Previous studies have shown that PFAS exposure disrupts the thyroid hormone balance although different results have been reported in different studies. A population-based cohort study by Lin et al., including 551 participants aged between 12 and 30 years, showed a positive association between PFNA levels and FT4 [15], as was found in present study. An analysis including 1181 participants of 20 years and older from NHANES 2007-2010 showed a positive association between PFOA and TT3 in women, a positive association between PFHxS and TT3 and TT4 in women and a negative association between PFHxS and FT4 in men [11]. An NHANES analysis including 1540 participants from 2007-2008 showed no significant association between PFAS and FT3 and FT4; PFOA was positively associated with TT3 and PFHxS was positively associated with TT4 [16]. Webster et al. included 1525 adult participants from the 2007-2008 NHANES cycle and only found a positive association between PFOA and FT3 in participants with normal iodine and thyroid peroxidase antibody levels. No significant associations between PFAS and thyroid hormones were found in participants with low iodine levels [10]. A cross-sectional analysis of 52,296 adults exposed to a year or more of PFAS (PFOA and PFOS) in drinking water and enrolled in the C8 Health Project showed significant elevations of TT4 and reductions in T3 uptake; no significant associations were found with TSH [29]. In 506 male employees enrolled in a fluorochemical medical surveillance program, no association was found between PFOA and TSH or TT4, while a negative association with FT4 and a positive association with TT3 were found [30]. Studies focusing on pregnant women have shown no association between PFAS and thyroid hormones [12,13], except for an increase in TSH associated with increased PFOS levels [13]. Moreover, there are some indications that exposure to certain other endocrine disrupting chemicals, including organochlorine pesticides and polychlorinated biphenyls, may be associated with an increased prevalence of autoimmune thyroiditis among exposed individuals [31,32]; however, future research is needed to investigate if this association also exists for PFAS.

PFAS may affect thyroid hormone levels via multiple mechanisms. PFAS may increase T4 metabolism in the liver and thyroid, replace T4 from serum binding proteins, and reduce T4 production in the thyroid gland. In vitro studies have, for instance, demonstrated that PFAS can compete with T4 for binding to transthyretin, a transport protein that binds approximately $15 \%$ of thyroid hormones, which is thought to then slowly increase circulating levels of FT4 [33]. In rats, PFOS has also been shown to increase T4 metabolism and excretion by upregulation of key hepatic transport proteins [34] and increasing T4 glucuronidation and excretion from the liver [35]. Furthermore, it has been shown in rats that PFAS can increase the conversion of $\mathrm{T} 4$ to $\mathrm{T} 3$ in the thyroid gland by upregulation of the de-iodinase enzyme DIO1 [36].

To date, no studies have investigated the effect of smoking on the association between PFAS exposure and thyroid hormones even though it has been shown in prior studies that smoking affects thyroid function and is inversely associated with thyroid cancer. Smoking is associated with a dose-dependent decrease in TSH in population-based studies [37]. This was demonstrated in a study using NHANES III including 15,592 participants, which showed that smoking appears to be positively associated with a mild TSH decrease [23]. These results were furthermore confirmed by a study including 5639 participants of the 2013-2015 Korean National Health and Nutrition Examination Survey, which found lower TSH levels in current smokers; this finding was even more apparent in iodine-deficient participants [38]. The mechanism by which smoking decreases TSH levels remains unknown [37]; lower TSH levels however may explain why PFAS exposure is not positively associated with FT4 in smokers as it is in non-smokers. Moreover, smokers have a higher prevalence of nontoxic goiter and multiple thyroid nodules, specifically in iodine-deficient areas [37], and smoking is inversely 
associated with thyroid cancer where the reduced risk is more pronounced for papillary compared to follicular thyroid cancer $[37,39,40]$. It has been hypothesized that lower BMI and lower TSH levels may contribute to this reduced cancer risk [37]; however, there are also some indications that genetic factors might play a role. A study has demonstrated an inverse association between germline CYP1A1 inheritance and smoking with risk of papillary thyroid cancer [41]. The results of the current study emphasize the complexity of the interaction between a variety of exposures and the thyroid gland and the need for future studies to investigate the effect of multiple diverse exposures, individually and combined, on thyroid function and thyroid cancer, especially because of the worldwide increase in thyroid cancer [42].

To investigate the association between PFAS exposure and thyroid hormones, we were only able to include the 2011-2012 NHANES lab subsample, in which participants had both PFAS and thyroid hormone measurements completed. Unfortunately, no adjacent sampling cycle measured both PFAS and thyroid hormones in the same lab subsample, therefore limiting our sample size. This study population is however a representative sample of the civilian non-institutionalized US population. Although we were able to adjust for important covariates in our analysis, we were unable to adjust for exposure to other endocrine disrupting chemicals (e.g., polychlorinated biphenyls (PCBs), organochlorines, dioxins, bisphenol A (BPA), polybrominated diphenyl ethers or phthalates), which have been associated with the disruption of thyroid function in prior studies [5], because these chemicals were not all measured in the same lab subsample. Another limitation is the cross-sectional nature of the data as both PFAS and thyroid hormone levels were measured at the same moment in time so reverse causation cannot be ruled out.

\section{Conclusions}

The current study is the first to show that the association between thyroid function, in particular FT4 levels, and PFAS exposure is modified by smoking in adults without a history of thyroid disease. These results may have implications for studies of other endocrine disrupting chemicals whose effects on the thyroid gland may potentially be modified by smoking as well. Furthermore, demonstrating effect modification by a second exposure has implications for future studies investigating the effects of multiple exposures on thyroid function, including smoking, especially for compounds acting via similar pathways.

Author Contributions: Conceptualization, M.v.G., E.T. and E.G.; formal analysis, M.v.G. and N.A.; writing—original draft, M.v.G., M.A. and K.Z.; writing—review and editing, M.v.G., N.A., M.A., K.Z., E.T. and E.G. All authors have read and agreed to the published version of the manuscript.

Funding: This research received no external funding.

Conflicts of Interest: The authors declare that they have no competing interest.

\section{References}

1. Fromme, H.; Tittlemier, S.A.; Volkel, W. Perfluorinated compounds-exposure assessment for the general population in Western countries. Int. J. Hyg. Environ. Health 2009, 212, 239-270. [CrossRef] [PubMed]

2. Giesy, J.P.; Kannan, K. Global distribution of perfluorooctane sulfonate in wildlife. Environ. Sci. Techol. 2001, 35, 1339-1342. [CrossRef] [PubMed]

3. Lau, C.; Anitole, K.; Hodes, C. Perfluoroalkyl acids: A review of monitoring and toxicological findings. Toxicol. Sci. 2007, 99, 366-394. [CrossRef] [PubMed]

4. Kato, K.; Wong, L.Y.; Jia, L.T. Trends in exposure to polyfluoroalkyl chemicals in the U.S. Population: 1999-2008. Environ. Sci. Technol. 2011, 45, 8037-8045. [CrossRef]

5. Gore, A.C.; Chappell, V.A.; Fenton, S.E. EDC-2: The Endocrine Society's Second Scientific Statement on Endocrine-Disrupting Chemicals. Endocr. Rev. 2015, 36, E1-E150. [CrossRef]

6. Bergman, Å.; Heindel, J.J.; Jobling, S. State of the Science of Endocrine Disrupting Chemicals 2012; World Health Organization: Geneva, Switzerland, 2013.

7. Kissa, E. Fluorinated Surfactants and Repellents; CRC Press: Boca Raton, FL, USA, 2001. 
8. US Environmental Protection Agency: Drinking Water Health Advisories for PFOA and PFOS. Available online: https://www.epa.gov/ground-water-and-drinking-water/drinking-water-health-advisoriespfoa-and-pfos (accessed on 10 September 2020).

9. IARC Monographs: Perfluorooctanoic Acid. Available online: https://monographs.iarc.fr/wp-content/ uploads/2018/06/mono110-01.pdf (accessed on 10 September 2020).

10. Webster, G.M.; Rauch, S.A.; Marie, N.S. Cross-Sectional Associations of Serum Perfluoroalkyl Acids and Thyroid Hormones in U.S. Adults: Variation According to TPOAb and Iodine Status (NHANES 2007-2008). Environ. Health Perspect. 2016, 124, 935-942. [CrossRef]

11. Wen, L.L.; Lin, L.Y.; Su, T.C. Association between serum perfluorinated chemicals and thyroid function in U.S. adults: The National Health and Nutrition Examination Survey 2007-2010. J. Clin. Endocrinol. Metab. 2013, 98, E1456-E1464. [CrossRef]

12. Webster, G.M.; Venners, S.A.; Mattman, A. Associations between perfluoroalkyl acids (PFAS) and maternal thyroid hormones in early pregnancy: A population-based cohort study. Environ. Res. 2014, 133, 338-347. [CrossRef]

13. Wang, Y.; Starling, A.P.; Haug, L.S. Association between perfluoroalkyl substances and thyroid stimulating hormone among pregnant women: A cross-sectional study. Environ. Health 2013, 12, 76. [CrossRef]

14. Lopez-Espinosa, M.J.; Mondal, D.; Armstrong, B. Thyroid function and perfluoroalkyl acids in children living near a chemical plant. Environ. Health Perspect. 2012, 120, 1036-1041. [CrossRef]

15. Lin, C.Y.; Wen, L.L.; Lin, L.Y. The associations between serum perfluorinated chemicals and thyroid function in adolescents and young adults. J. Hazard. Mater. 2013, 244-245, 637-644. [CrossRef] [PubMed]

16. Jain, R.B. Association between thyroid profile and perfluoroalkyl acids: Data from NHANES 2007-2008. Environ. Res. 2013, 126, 51-59. [CrossRef] [PubMed]

17. Melzer, D.; Rice, N.; Depledge, M.H. Association between serum perfluorooctanoic acid (PFOA) and thyroid disease in the U.S. National Health and Nutrition Examination Survey. Environ. Health Perspect. 2010, 118, 686-692. [CrossRef] [PubMed]

18. Winquist, A.; Steenland, K. Perfluorooctanoic acid exposure and thyroid disease in community and worker cohorts. Epidemiology 2014, 25, 255-264. [CrossRef] [PubMed]

19. Steenland, K.; Fletcher, T.; Savitz, D. C8 Science Panel Status Report: PFOA and Adult Thyroid Disease in the Mid-Ohio Valley. 2011. Available online: http://www.c8sciencepanel.org/pdfs/Status_Report_C8_and_ thyroid_disease_5Dec2011.pdf (accessed on 10 September 2020).

20. Barry, V.; Winquist, A.; Steenland, K. Perfluorooctanoic acid (PFOA) exposures and incident cancers among adults living near a chemical plant. Environ. Health Perspect. 2013, 121, 1313-1318. [CrossRef]

21. Cho, C.R.; Lam, N.H.; Cho, B.M. Concentration and correlations of perfluoroalkyl substances in whole blood among subjects from three different deographical areas in Korea. Sci. Total Environ. 2015, 512-513, 397-405. [CrossRef]

22. Soldin, O.P.; Goughenour, B.E.; Gilbert, S.Z. Thyroid hormone levels associated with active and passive cigarette smoking. Thyroid 2009, 19,817-823. [CrossRef]

23. Belin, R.M.; Astor, B.C.; Powe, N.R. Smoke exposure is associated with a lower prevalence of serum thyroid autoantibodies and thyrotropin concentration elevation and a higher prevalence of mild thyrotropin concentration suppression in the third National Health and Nutrition Examination Survey (NHANES III). J. Clin. Endocrinol. Metab. 2004, 89, 6077-6086.

24. Kuklenyik, Z.; Needham, L.L.; Calafat, A.M. Measurement of 18 perfluorinated organic acids and amides in human serum using on-line solid-phase extraction. Anal. Chem. 2005, 77, 6085-6091. [CrossRef]

25. Graber, J.M.; Alexander, C.; Laumbach, R.J. Per and polyfluoroalkyl substances (PFAS) blood levels after contamination of a community water supply and comparison with 2013-2014 NHANES. J. Expo. Sci. Environ. Epidemiol. 2019, 29, 172-182. [CrossRef]

26. Garber, J.; Cobin, R.; Gharib, H. Clinical practice guidelines for hypothyroidism in adults: Cosponsored by the American Association of Clinical Endocrinologists and the American Thyroid Association. Endocr. Pract. 2012, 18, 988-1028. [CrossRef] [PubMed]

27. Benowitz, N.L.; Bernert, J.T.; Caraballo, R.S. Optimal serum cotinine levels for distinguishing cigarette smokers and nonsmokers within different racial/ethnic groups in the United States between 1999 and 2004. Am. J. Epidemiol. 2009, 169, 236-248. [CrossRef] [PubMed] 
28. Buttke, D.E.; Sircar, K.; Martin, C. Exposures to endocrine-disrupting chemicals and age of menarche in adolescent girls in NHANES (2003-2008). Environ. Health Perspect. 2012, 120, 1613-1618. [CrossRef] [PubMed]

29. Knox, S.S.; Jackson, T.; Frisbee, S.J. Perfluorocarbon exposure, gender and thyroid function in the C8 Health Project. J. Toxicol Sci. 2011, 36, 403-410. [CrossRef] [PubMed]

30. Olsen, G.W.; Zobel, L.R. Assessment of lipid, hepatic, and thyroid parameters with serum perfluorooctanoate (PFOA) concentrations in fluorochemical production workers. Int. Arch. Occup. Environ. Health 2007, 81, 231-246. [CrossRef] [PubMed]

31. Ferrari, S.M.; Fallahi, P.; Antonelli, A. Environmental issues in thyroid diseases. Front. Endocrinol. 2017, 8. [CrossRef]

32. Benvenga, S.; Elia, G.; Ragusa, F.; Paparo, S.R.; Sturniolo, M.M.; Ferrari, S.M.; Antonelli, A.; Fallahi, P. Endocrine disruptors and thyroid autoimmunity. Best Pract. Res. Clin. Endocrinol. Metab. 2020, 34, 101377. [CrossRef]

33. Weiss, J.M.; Andersson, P.L.; Lamoree, M.H. Competitive binding of poly- and perfluorinated compounds to the thyroid hormone transport protein transthyretin. Toxicol. Sci. 2009, 109, 206-216. [CrossRef]

34. Yu, W.G.; Liu, W.; Liu, L. Perfluorooctane sulfonate increased hepatic expression of OAPT2 and MRP2 in rats. Arch. Toxicol. 2011, 85, 613-621. [CrossRef]

35. Chang, S.C.; Thibodeaux, J.R.; Eastvold, M.L. Thyroid hormone status and pituitary function in adult rats given oral doses of perfluorooctanesulfonate (PFOS). Toxicology 2008, 243, 330-339. [CrossRef]

36. Yu, W.G.; Liu, W.; Jin, Y.H. Effects of perfluorooctane sulfonate on rat thyroid hormone biosynthesis and metabolism. Environ. Toxicol. Chem. 2009, 28, 990-996. [CrossRef] [PubMed]

37. Wiersinga, W.M. Smoking and thyroid. Clin. Endocrinol. 2013, 79, 145-151. [CrossRef] [PubMed]

38. Park, S.; Kim, W.G.; Jeon, M.J.; Kim, M.; Oh, H.-S.; Han, M.; Kim, T.Y.; Shong, Y.K.; Kim, W.B. Serum thyroid-stimulating hormone levels and smoking status: Data from the Korean National Health and Nutrition Examination Survey VI. Clin. Endocrinol. 2018, 88, 969-976. [CrossRef] [PubMed]

39. Cho, Y.A.; Kim, J. Thyroid cancer risk and smoking status: A meta-analysis. Cancer Causes Control. 2014, 25, 1187-1195. [CrossRef]

40. Mack, W.J.; Preston-Martin, S.; Dal Maso, L. A pooled analysis of case-control studies of thyroid cancer: Cigarette smoking and consumption of alcohol, coffee, and tea. Cancer Causes Control. 2003, 14, 773-785. [CrossRef]

41. Bufalo, N.E.; Leite, J.L.; Guilhen, A.C. Smoking and susceptibility to thyroid cancer: An inverse association with CYP1A1 allelic variants. Endocr. Relat. Cancer 2006, 13, 1185-1193. [CrossRef]

42. Davies, L.; Welch, H.G. Increasing incidence of thyroid cancer in the United States, 1973-2002. JAMA 2006, 295, 2164-2167. [CrossRef]

Publisher's Note: MDPI stays neutral with regard to jurisdictional claims in published maps and institutional affiliations.

(C) 2020 by the authors. Licensee MDPI, Basel, Switzerland. This article is an open access article distributed under the terms and conditions of the Creative Commons Attribution (CC BY) license (http://creativecommons.org/licenses/by/4.0/). 